\title{
Sind niedrige Vitamin-D-Spiegel mit höheren MS-Schubraten assoziiert?
}

Fragestellung: Gibt es einen Zusammenhang zwischen niedrigen Vitamin-D-Spiegeln und einer ansteigenden Schubrate bei Patienten mit schubförmiger Multipler Sklerose (MS)?

Hintergrund: Mehrere Studien und Fallberichte gaben Hinweise auf einen möglichen Zusammenhang zwischen dem VitaminD-Serumspiegel und einer erhöhten Erkrankungsaktivität bei Patienten mit schubförmig verlaufender MS. Die bisherigen Studien waren jedoch relativ klein und widersprüchlich. Ein weiteres Problem der Vitamin-D-Studien sind nicht standardisierte Testverfahren zur Spiegelmessung sowie die Frage, welcher Vitamin-D-Metabolit der geeignetste für Studien dieser Art ist.

Runia TF, Hop WC, de Rijke YB et al. Lower serum vitamin $D$ levels are associated with a higher relapse risk in multiple sclerosis. Neurology 2012; 79: 261-6
Patienten und Methoden: In einer prospektiven longitudinalen Studie an 73 Patienten mit schubförmiger MS wurden Blutspiegel der 25-Hydroxy-Vitamin-D (25-OH-D) alle acht Wochen gemessen. Ein möglicher Zusammenhang zwischen den 25-OH-D-Spiegeln und Schubraten wurde durch das „Poisson Regressions Model“ mit den individuellen Serumspiegeln als zeitabhängige Variable berechnet.

Ergebnisse: Während der Beobachtungsphase (im Durchschnitt 1,7 Jahre) erlitten 58 Patienten insgesamt 139 Schübe. Die 25-OHD-Spiegel wurden kategorisiert in niedrig $(<50 \mathrm{nmol} / \mathrm{l})$, mittel $(50-100 \mathrm{nmol} / \mathrm{l})$ und hoch (> $100 \mathrm{nmol} / \mathrm{l})$. Das monatliche Schubrisiko verringerte sich signifikant im Vergleich zur Gruppe der niedrigen 25-OH-D-Spiegel auf 0,7 und 0,5 in den Gruppen mit mittleren und mit höheren Vitamin-D-Spiegeln $(p=0,007)$. Der Zusammenhang zwischen 25-OH-D-Spiegeln und der Schubrate war linear ohne erkennbare Grenze. Mit der jeweiligen Verdopplung der 25-OH-D-Serumspiegel verringerte sich die Schubrate um $27 \%$ (95\%-Konfidenzintervall $8-42 \%, \mathrm{p}=0,008)$.

Schlussfolgerungen: Die Ergebnisse dieser Studie zeigen einen klaren Zusammenhang zwischen hohen Vitamin-D-Spiegeln und einer reduzierten Schubrate bei Patienten, die unter einem schubförmigen Verlauf der MS leiden. Die Studie zeigt damit einen klaren Effekt von Vitamin D auf den Erkrankungsverlauf beziehungsweise die Erkrankungsaktivität bei schubförmiger MS.

\section{-Kommentar von Volker Limmroth, Köln-Merheim}

\section{Vitamin D ist doch relevant}

Hier also eine weitere, leider kleine Studie mit circa 70 Patienten, die allerdings im Hinblick auf das Studiendesign wenig kritisiert werden kann. Die holländischen Autoren haben nicht den aktiven Metaboliten des Vitamin-D (1,25-dehydroxy-Vitamin-D = 1,25-di O-D) gewählt, sondern den Metaboliten 25$\mathrm{OH}-\mathrm{D}$, der den Vitamin-D-Status des Patienten wahrscheinlich am besten reflektiert. Sie hatten ferner die mühsame, zweimonatliche Spiegelkontrolle gewählt, um jahreszeitliche Effekte, die bekanntermaßen Auswirkungen auf den Vitamin-D-Spiegel haben können, besser zu erfassen und entsprechend zu berücksichtigen. Die Studie ist damit erst die zweite prospektiv

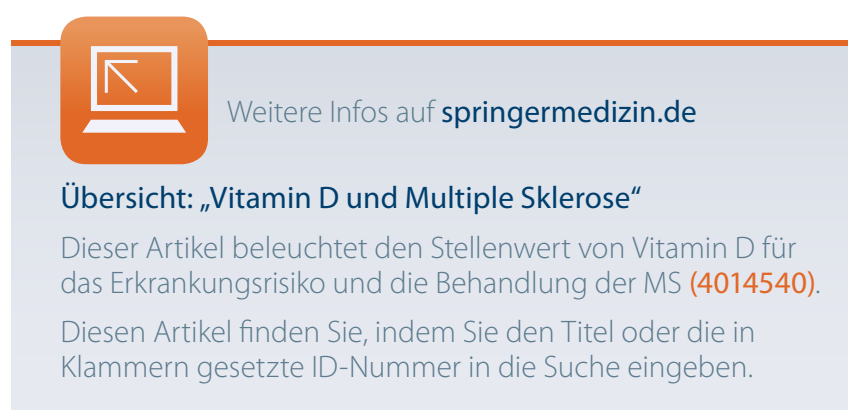

durchgeführte Studie zu dieser Thematik, die sich ferner durch den Aspekt auszeichnet, dass nur eine kleine Gruppe von Patienten unter einer immunmodulatorischen Dauertherapie mit Interferon stand, sodass die Studie im Wesentlichen die mögliche Wirkung von Vitamin D - unabhängig von einer immunmodulatorischen Grundbehandlung - untersuchen konnte.

Zusammenfassend handelt es sich hier um wichtige und solide Ergebnisse, die eine Rolle des Vitamin D stützen. Dennoch müsste vor allgemeinen Empfehlungen zur großfächigen Einnahme von Vitamin D nun doch eine prospektive randomisierte Studie mit einer deutlich größeren Studienpopulation durchgeführt werden.

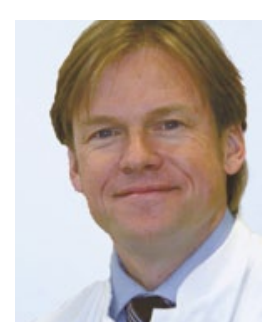

Prof. Dr. med. Volker Limmroth, Köln-Merheim

Chefarzt der Klinik für Neurologie und Palliativmedizin Köln-Merheim E-Mail: LimmrothV@kliniken-koeln.de 